\title{
Reducing the parasitic inductance of the meander resistive element in a powerful block of brake resistors of electric vehicles
}

\author{
Anton Timofeev ${ }^{1, *}$ and Alexander Shapovalov ${ }^{2}$ \\ ${ }^{1}$ Research and production company «Etna plus», 410040, Vishnevaya str. 11, Saratov, Russia \\ ${ }^{2}$ Saratov State University, Physical Department, 4120012. Astrakhanskaya str. 83, Saratov, Russia
}

\begin{abstract}
The dependence of the magnetic properties and inductance of the braking resistors block of electric transport on his configuration and main dimensions of the tape resistive elements included in it and their relative position is investigated. Approaches to reducing spurious inductance of various configurations of a powerful block of brake resistors for subway trains had been found.
\end{abstract}

\section{Introduction}

In many devices, including in public urban and railway transport, crane equipment and other mechanisms, there are operating modes in which electric motors go into generation mode, for example, when electrodynamic braking of electric transport is carried out. For safely discharge the engine generation energy, as a rule, the so-called blocks of brake resistors are used, in which the braking energy is converted into thermal energy dissipated in the surrounding space $[1,2]$.

In this paper, we consider ribbon meander resistors for subway cars and electric trains (Fig. 1), consisting of a fechral tape, curved into a meander with a cross section of $60 * 1,2$ $\mathrm{mm}$ and a resistance of $0,44 \div 0,52 \mathrm{Ohm}$ (depending on the resistors operation temperature), through which current flows up to a thousand or more amperes $[3,4]$.

These resistors must have a minimum inductance value. This is due to the fact that devices of this type work in conjunction with inverter electronic motor controllers operating in the PWM-switched load mode with a reference frequency of the order of several tens of $\mathrm{kHz}$ and a current in the form of rectangular pulses with a duration of less than $1 \mathrm{~ms}$, flows through them [1]. In such conditions, in order to increase the efficiency of interaction between the elements of the "network-controller-motor-brake resistor" system and minimize the interference it creates, it is desirable to have the resistor blocks inductance, as little as possible. In the case, considered in this paper, the resistor inductance value must be limited at the level of $10 \mu \mathrm{H}$.

The task, therefore, is to minimize the inductance under certain restrictions, which are the temperature (which should not exceed the specified value) and a rather narrow range of temperature changes in the resistance within the limits above.

\footnotetext{
${ }^{*}$ Corresponding author: anton_timofeev@list.ru
} 


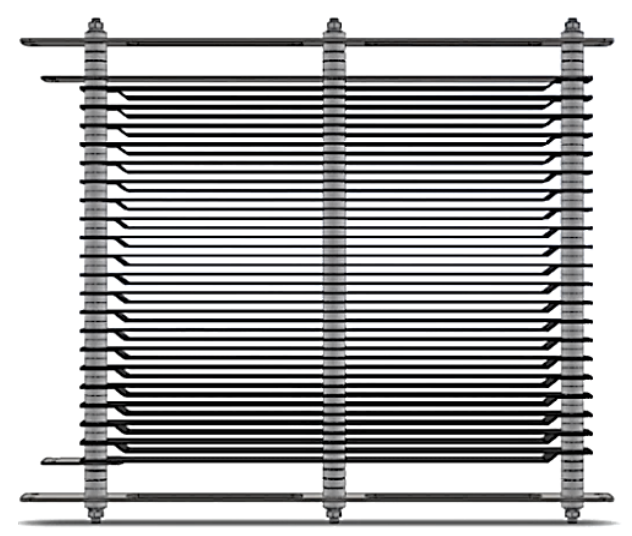

a

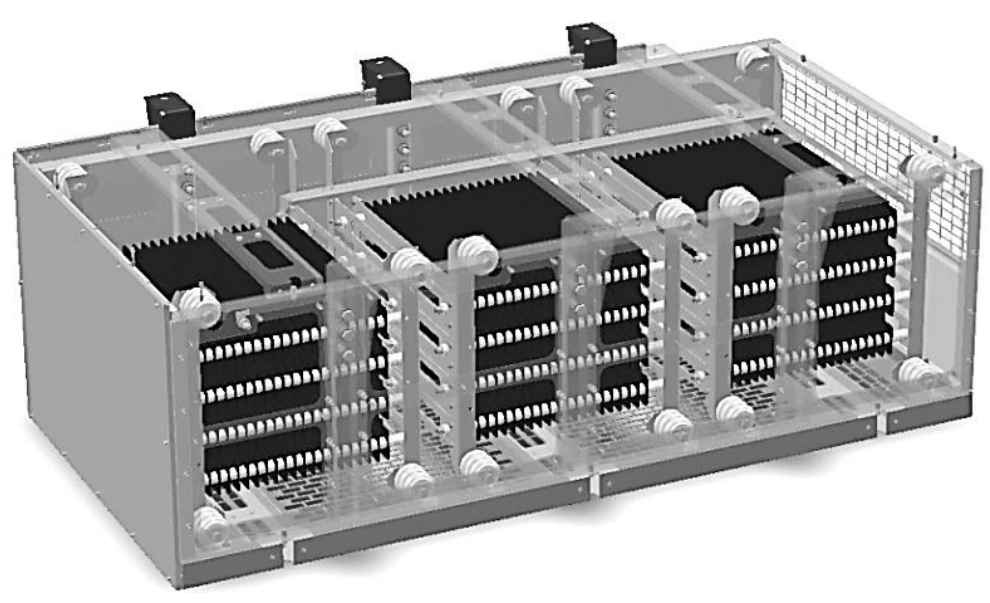

b

Fig. 1. Appearance of tape resistive elements (a) and a block of brake resistors for subway cars with open top and side walls (b)

In this case, the temperature is determined, on one hand, by the heat dissipation in the unit - due to the conversion of braking energy into heat in it (Fig. 2 shows a typical time dependence of energy release during braking for this type of device). On the other hand, the temperature regime is determined by the parameters of heat removal from the working (heat-dissipating) part of the block of brake resistors.

Due to the design features of these devices' placement in cars of urban and railway transport (resistor blocks are often placed in the space under the car), it is not possible to implement forced heat removal in them, as well as heat removal by the air flow that is imposed when the vehicle is moving. Therefore, heat dissipation is often organized on the basis of the "stove draft" principle.

To ensure this, it should be created a structure from resistive elements (Fig. 1), that is sufficiently massive and maximally "transparent" for air in the vertical direction, in which the kinetic energy supplied during braking (which is already converted into engine generation electrical energy), is converted into the heat energy, increasing the temperature of the resistive elements to several hundred degrees.

In this case, due to the temperature difference between the environment and the resistive elements (and heating up from them the adjacent areas of air, filling the free space of the 


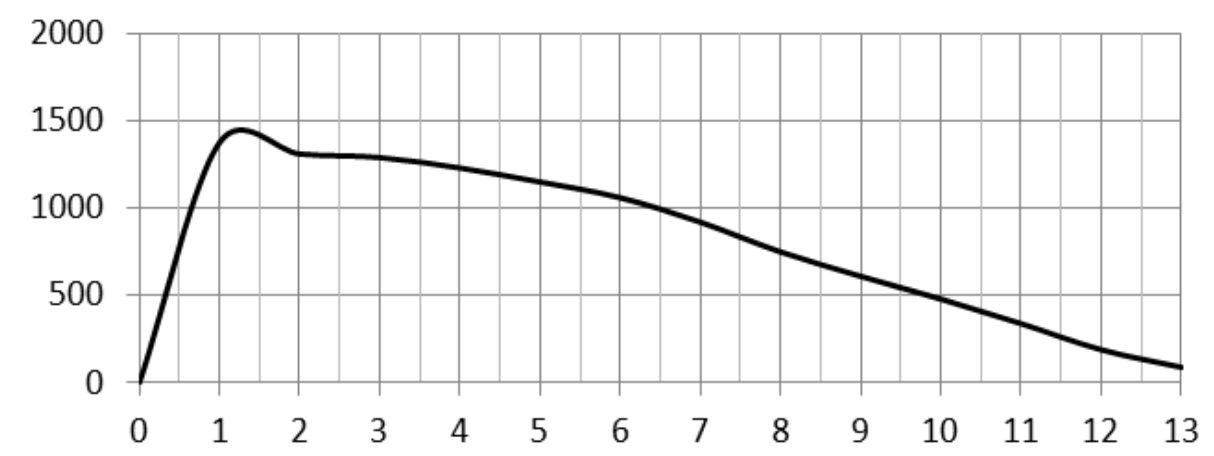

Fig. 2. A typical graph of the thermal energy release $(\mathrm{kW})$ in a resistor unit during a braking cycle versus time (s).

block resistors), the air flow, passing through the resistor in the vertical direction, occurs. The value of the air flow (flowing through the resistor) $Q[\mathrm{~m} 3 / \mathrm{s}]$ is determined by the "stove draft" formula [5]:

$$
Q=C A \sqrt{2 g H \frac{T_{i}-T_{0}}{T_{i}}}
$$

where A - is the horizontal cross-sectional area, $H$ - is the height of the air channel, $C$ - is the coefficient introduced due to friction $(\sim 0.7) ; g$ - gravitational acceleration, $T_{i}, T_{o}$ average internal and absolute external temperatures.

In practice, the horizontal section of the "stove draft" channel varies in height in such structures, so it is necessary to choose a certain average (effective) section of channel $A$, and substitute it in formula (1).

Thus, the temperature is determined by the configuration and flow value (1), and for its determination it is necessary to solve the nonlinear thermophysical problem. The considered brake resistor for subway cars has an average power dissipation of $785 \mathrm{~kW}$, a braking pulse duration of $13 \mathrm{~s}$ (Fig. 2), and a maximum power dissipation of $\geq 1,4 \mathrm{MW}$.

The real cycle of its operation is as follows: $13 \mathrm{~s}$ - braking (energy release in accordance with the schedule in Fig. 2), then $25 \mathrm{~s}$ - stop and $90 \mathrm{~s}$ - movement between stations (total time in the cooling mode - $115 \mathrm{~s}$ ). In this block, there are 3 resistors connected in parallel, each of which consists of 4 sequentially connected tape resistive elements (TRE), mounted one above the other to organize heat removal according to the "stove draft" principle. Such TRE were used, in particular, while creating a block of braking resistors for metro cars (Fig. 1b).

The task is to consider the dependences of the inductance of such devices on the main dimensions and configuration, and on the relative position of the resistive elements included in them. The review is carried out in relation to the developed design of brake resistor blocks, in which tape resistors are used as resistive elements [4].

\section{The calculation of the brake resistors block inductance}

The selected configuration of resistive elements, from series-parallel connection of which the braking resistor architecture is built, is shown in Fig. 3. The main characterizing it parameters are: the meander height (span) $l$, the pitch of this periodic structure $h$, the tape thickness $t$ and its width $w$. 


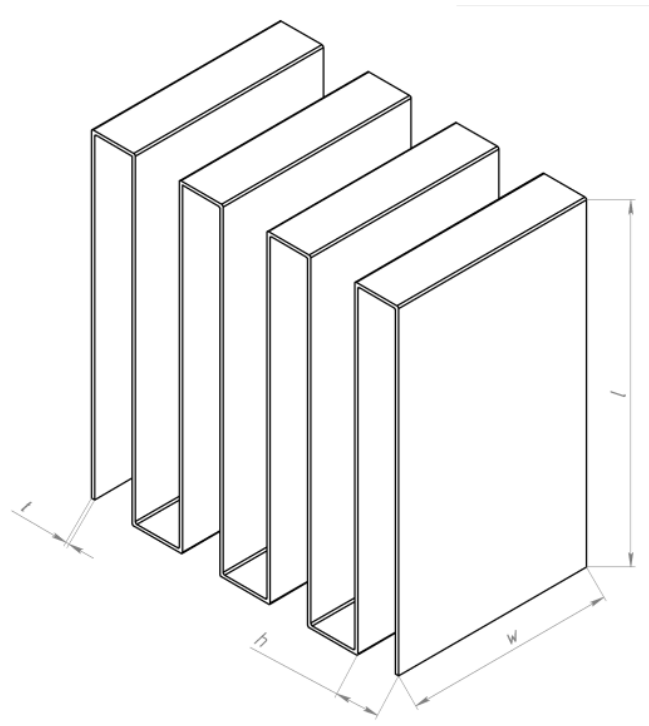

Fig. 3. Appearance of a meander resistive element.

In the case of a large number of periods, a meander line with a direct current has the property that, when there is a large meander span $l$ between adjacent lines and due to the opposite direction of currents (Fig. 4), an electric wall can be installed between them. Therefore, the inductance of one tape of length $l$ will be equal to the linear inductance of the strip line in the screen $L_{0}$ multiplied by $l$. The composition of the linear inductance and the linear capacity is the inversely proportional to the squared speed of light.

For wave resistance and strip line capacitance in the screen, formulas written through complete elliptical integrals take place.

However, in our case of a wide tape (with a width of $w<<t$ and $w<<h$, where $t$ and $h$ are respectively the thickness of the tape and half the period $h=d / 2$ ), with a very high degree of accuracy $C_{0}=2 \varepsilon_{0} \mathrm{w} / \mathrm{h}$ can be used as the linear capacity. Adding to it a boundary capacitance, we will find the total linear inductance $L_{0}$. Considering the inductance of the shoulders (fig. 3), we can find the total inductance of one section:

$$
L(N, l, w, h, t)=\frac{N l \mu_{0}}{4 w /(h-t)+t / h}+\frac{N \mu_{0} h}{2 \pi} \ln (h /(w+t)+1 / 2)
$$

Here $N$ - is the number of periods (sections), $l$ - is the meander span, $w, t$ - are the transverse dimensions of the tape.

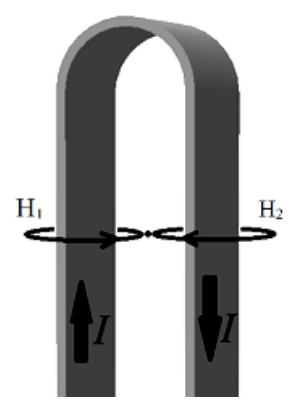

Fig. 4. Brake resistor tape element and current direction. 


\section{The solution of the problem of reducing the resistive elements of the block inductance}

The tasks of reducing the inductance can be performed using the procedure of minimizing the function (2) under the imposition of conditions for finding the resistance and temperature in the given limits. The inductance reduction is also based on a series-parallel circuit of connecting the meanders in the resistor block (Fig. 1). This connection allows to create a three-dimensional block of resistors with the required stove draft. The resistance $R$ of a single meander is proportional to the length of the fechral tape $N(l+d)$ formed into it and inversely proportional to its cross-section area $w t$.

Taking into account the dependence of the resistivity on temperature, for a given current value, the specific power density of the sources was calculated and the nonlinear heat problem was solved taking also into account convection heat transfer and the air flow (1). Design optimization was carried out iteratively. Firstly, the inductances were calculated for a given temperature-independent resistance.

For the device under study, the calculation and actual measurement of the inductance was carried out, based on which the conclusion was made: the magnetic fields of adjacent tapes connected in series to each other by TRE, as well as parallel-connected groups of resistors located in the structure at a sufficiently large distance from each other, practically do not affect each other. Therefore, the inductance of any of these systems [6] can be calculated using the formula

$$
\widetilde{L}=L(N, l, w, h) n / m
$$

where $N$ - is the number of bands (half-periods) in the TRE, $n$ - is the number of TREs connected in series to each other, $m$ - is the number of resistor groups connected in parallel (from series-connected TREs).

This made it possible to use this formula for calculating other configurations of the block of brake resistors shown below in Table 1 (any of the given configurations during the tests provided almost identical values of the total resistance of the brake resistors block about $0.5 \Omega$ ).

Table 1. Configuration of brake resistor blocks

\begin{tabular}{|c|c|c|c|}
\hline $\begin{array}{c}\text { Ribbon } \\
\text { section, mm }\end{array}$ & $\begin{array}{c}\text { The number of } \\
\text { parallel bands in the } \\
\text { TRE (n) }\end{array}$ & $\begin{array}{c}\text { The number of } \\
\text { consecutively connected } \\
\text { TRE in resistor (k) }\end{array}$ & $\begin{array}{c}\text { The number of parallel } \\
\text { united resistors in } \\
\text { block (m) }\end{array}$ \\
\hline $0,6^{*} 60$ & 41 & 2 & 3 \\
\hline $0,9 * 60$ & 41 & 3 & 3 \\
\hline $1,2 * 60$ & 41 & 4 & 3 \\
\hline $1,5 * 60$ & 41 & 5 & 3 \\
\hline
\end{tabular}

Since the inductance and resistance of series-parallel connecting have the same dependence on $n$ and $m$, it is convenient to minimize formula (3). On the basis of formulas (1), (2), (3), a program was constructed in the Matcad programming system, according to which the TRE temperature growth curves were obtained (Fig. 5) in the mode of sequential braking with heat generation and cooling the TRE until reaching a stationary temperature. Corresponding time dependences of temperature for multiple cycles of power release and cooling of resistive elements were constructed for several different cross-sections of the "stove draft pipe" (Fig. 5). 


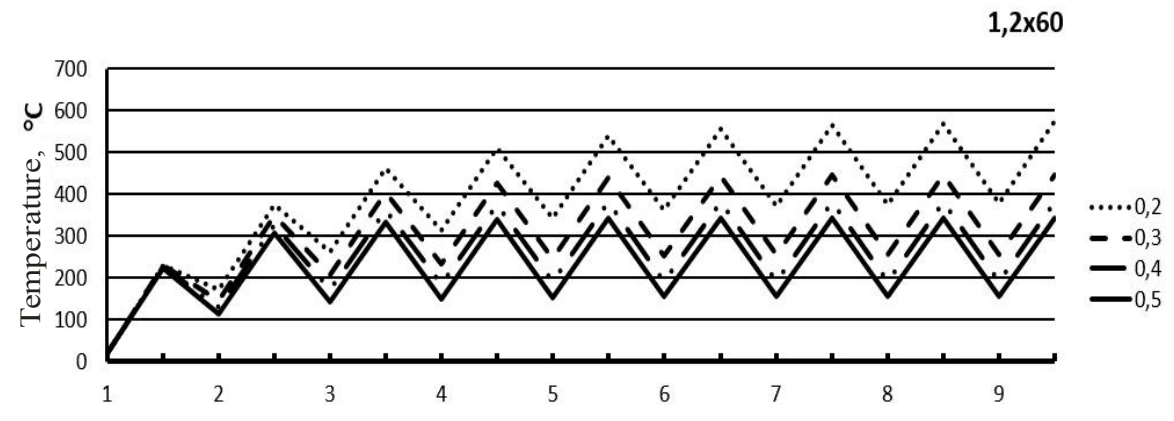

\section{Number of deceleration cycles}

Fig. 5. Quasi-stationary temperature exit curves for resistive elements made of fechral tape with a cross section of $1,2 * 60 \mathrm{~mm}^{2}$ (the numbers of heating and cooling cycles of resistive elements are shown at the abscissa scale).

The nonlinear optimization problem should be solved now, taking into account minimization (1) with the given restrictions: $\min \left[\Phi\left(N, l, w, h, t, \lambda_{1}, \lambda_{2}, \lambda_{3}\right)\right]$, where

$$
\begin{aligned}
& \Phi\left(N, l, w, h, t, \lambda_{1}, \lambda_{2}\right)=L(N, l, w, h, t)+\lambda_{1}\left|R(N, l, w, h, t, \rho(T))-R_{0}\right| \\
& +\lambda_{2}\left|T-T_{i}\right|+\lambda_{3}\left|Q\left(T_{0}, T, H, S,\right)-Q_{0}\right|
\end{aligned}
$$

and the Lagrange multipliers $\lambda 1, \lambda 2, \lambda 3$ are introduced.

Here $T_{i}$ - is the set value of the stationary temperature at the considered mode of transport operation. Unfortunately, the determination of temperature is a nonlinear problem requiring a numerical solution; therefore, analytical minimization (4) is impossible. Such minimization is possible only without taking into account the temperature and determines only preliminary values of the block parameters. The further optimization is possible based on iterative procedures. Since the cross-sectional area in (1) is determined as $S=N l h$, it is convenient to specify it as well as the value $H$.

Since the cross-sectional area in (1) is defined as $S=N l h$, it is convenient to set it in the same way as the value of $\mathrm{H}$. Hence, we have a relation $l=S /(\mathrm{Nh})$.

Considering the resistance without taking into account the temperature, we have $R\left(N, l, w, h, t, \rho_{0}\right)=\rho_{0} N l /(w t)=0.5 \mathrm{Ohm}$. Here, $\rho_{0}=0.0013 \mathrm{Ohm} \cdot \mathrm{m}$ - is the specific resistance of fechral at room temperature. And from here we can get the connection $l=0.5 w t /\left(N \rho_{0}\right)=S /(N h)$. So, $h=\rho_{0} S /(0.5 w t)$.

Substituting these relations in (2), we minimize the inductance in two variables by solving the equations $\partial_{w} L(N, l, w, h, t)=0, \partial_{t} L(N, l, w, h, t)=0$ for different integer values of $N$. The indicated approach allowed to obtain the optimal values of the width and thickness of the tape, as well as to choose the number of meander periods and block sizes. The best values are $N \sim 40, w \sim 60 \mathrm{~mm}$ and $t \sim 1 \div 1,5 \mathrm{~mm}$. The refinement of the parameters taking into account the temperature dependence of the specific resistance $\rho$ of fechral and temperature $T_{i}$ is possible based on the Lagrange function (4).

\section{Conclusion}

As a result of the research carried out in this paper, approaches have been developed to solve the problem of reducing of the brake resistors block inductance up to values, that 
allow to provide the efficient operation of the electric drives (with which they are used in vehicle electrical systems).

It should also be noted the high technology, constructive simplicity and heat removal efficiency (due to the creation of strong air draft) in the meander braking resistors, as well as the simplicity and good accuracy of determining their inductance.

\section{References}

1. Pat. for invention No. IZ2570923 Ros. Federation. Block of self-ventilating resistors for electric public transport / Kozlov A.V., Timofeev A.I., Grigoryan S.V. and etc; patent holder - NPF Etna. - declared. 07.08.2014, issue 11.18.2015, publ. 12.20.2015, Bull. Number 35

2. Pat. Utility Model No. PM145666 Ros. Federation. / Block of resistors for public transport / Kozlov A.V., Timofeev A.I.; patent holder - NPF Etna. - declared. 07.08.2014, issue 08.21.2014, publ. 10.10.2014, Bull. Number 21.

3. I. A. Kornev, A. I. Timofeev, A. S. Shapovalov. Analysis of thermophysical processes occurring in the braking resistor blocks of mobile electric vehicles // Scientific discussion: questions of technical sciences. No. 4 (17) ": Collection of articles on the materials of the XXI international correspondence scientific-practical conference. - M.: Internauka. P. 122-131 (2014)

4. Pat. Utility Model No. PM186566 Ros. Federation. Power resistor block / Timofeev A.I., Mashkov I.V. and etc.; patent holder of NPF Etna Plus, 10/10/2018, issue 01.24.2019, publ. 01.24.2019, Bull. Number 3.

5. N.P. Vintonyak, V.D. Semenov, V.A. Kabirov et al. Estimation of parasitic inductance of current-carrying tires in the power supply of the test complex for loading automatic DC circuit breakers // TUSUR Reports, Volume 19, No. 4. P. 129-132 (2016)

6. Kalantarov P.L., Zeitlin L.A. Inductance calculation. Ltningrad: Energoatomizdat, 488 p. (1986) 